\title{
Achondroplasia and spinal cord lesion. Three case reports
}

\author{
N Hamamci MD, * S Hawran MD, F Biering-Sørensen MD PhD \\ Centre for Spinal Cord Injured, Rigshospitalet, National University Hospital, \\ Copenhagen, Denmark.
}

The most important complications encountered by achondroplastic dwarfs are neurological problems related to a narrowed spinal canal. Stenosis of the spinal canal is secondary to abnormalities of endochondral ossification with premature synostosis of the ossification centres of the vertebral body and the posterior arch, resulting in thickening of the lamina, shortening of the pedicles, and reduced height of the vertebral bodies. Additional factors such as prolapsed intervertebral discs, osteophytes and progressive thoracolumbar kyphosis contribute to the narrowing of the spinal canal.

Three achondroplastic dwarfs having spinal stenosis and spinal cord lesion representing typical clinical courses are described.

Keywords: achondroplasia; dwarfism; spinal cord injury; spinal canal stenosis.

\section{Introduction}

Achondroplasia, the most common form of osteochondrodysplasia, is autosomal dominant with most cases being new mutations. It is characterised by rhizomelic shortness of the limbs, midface hypoplasia and defective endochondral bone development. ${ }^{1-3}$

There is a high incidence of neural complications associated with this form of dwarfism. ${ }^{4}$ Neural complications are present in about $50 \%$ of the patients and include hydrocephalus, hypoacusia, strabismus and brainstem damage due to the anomalous growth of the chondrocranium, peripheral nerve trunk damage as a result of the skeletal alterations and spinal cord injury (SCI) caused by diminished size of the spinal canal while the spinal cord and the cauda equina are of normal size. Normal vertebral development is the result of endochondral ossification, while in achondroplasia the morphological alterations of the spinal column are due to premature synostosis of the ossification centres of the vertebral body and posterior arch. The laminae are thickened, the pedicles short and stumpy

\footnotetext{
*On leave from Ankara Rehabilitation Centre, Ankara, Turkey.

Correspondence: Fin Biering-Sørensen, Centre for Spinal Cord Injured, Rigshospitalet, Havnevej 25, DK-3100 Hornbæk, Denmark.
}

and the vertebral bodies reduced in height. Thus the spinal canal is narrowed both anteroposteriorly and transversely (Fig 1). The spinal subarachnoid space is further reduced because the vertebral bodies are concave in their posterior aspect with the upper and lower surfaces projecting into the space of the vertebral canal. ${ }^{3}$

This report describes three achondroplastic dwarfs suffering from spinal stenosis and incomplete SCI who were admitted to our centre within a period of 3 years. Their histories illustrate some typical courses for this very special population of SCI patients.

\section{Case reports}

\section{Case 1}

A 45 year old, male, achondroplastic dwarf was admitted to the centre suffering from incomplete paraplegia. Six years previously he had compression fractures of vertebrae T12 and L1 resulting from a fall. At that time he was neurologically normal and was treated conservatively. Six months later he began to experience intermittent low back pain radiating bilaterally down to the feet. Three years later intermittent urinary retention and problems with constipation occurred, and another 2 years later he noted a gradual onset of bilateral leg weakness. This progressed to marked weakness with difficulty in ambulation. There was decreased sensation to pinprick and light touch below L2 


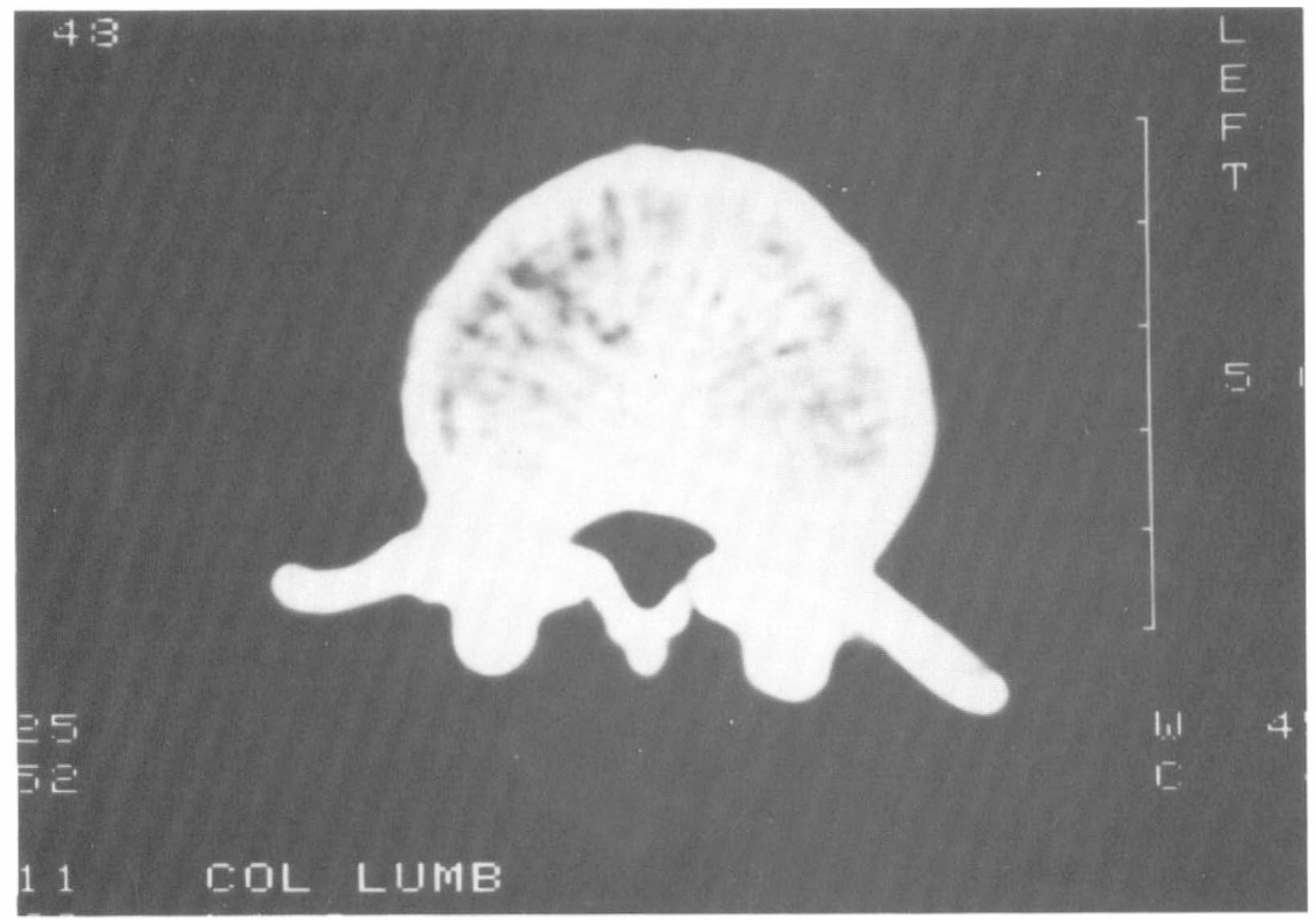

Figure 1 Severe spinal stenosis at level of vertebra L2 in a patient with achondroplasia and incomplete paraplegia (case 2).

bilaterally, his motor score ${ }^{5}$ was 35 in the right and 42 in the left side. CT scan showed a very severe spinal stenosis from vertebrae T10 to L3 and facet arthrosis. He was treated operatively by a decompressive laminectomy of vertebrae T12-L1-L2 and hemilaminectomy of vertebra T11. Postoperatively motor scores were right/ left $34 / 40$ and bladder emptying difficulty was present. Intermittent catheterisation was initiated, and 4 weeks after the operation he was transferred to our centre.

He was a small man having typical achondroplasia characteristics with a thoracolumbar kyphosis. He was unable to stand up alone without support. There were paresthesiae bilaterally over the lateral side of the femurs, lower legs and the feet. The motor scores were unchanged. Spasticity was present in both lower extremities.

During the rehabilitation process progress occurred. On discharge he was able to walk independently with one elbow crutch and was able to void normally. He still had problems with constipation.

\section{Case 2}

A 31 year old man, with achondroplasia and incomplete paraplegia was admitted to the centre.

When he was 26 years old he noted weakness in dorsiflexing his left foot and difficulty in walking. He was hospitalised and on neurolgical examination sensation was normal, there was paralysis and atrophy of the left ankle dorsiflexors, otherwise the motor power was normal in the lower extremities. Bladder and bowel functions were normal. Myelography showed a complete block of contrast at the level of vertebra L1. CT scan showed severe spinal stenosis (Fig 1) from vertebra T11 to L5 and a left lateral herniated disc at L2/3. He was treated conservatively and on discharge he was able to walk $1 \mathrm{~km}$ with a left ankle foot orthosis.

Five years later he had an acute onset of progressive low back pain without radiation to the legs, and 2 weeks later he was hospitalised because of the sudden development of flaccid paralysis of the lower extremities and urinary 
retention. There was hypoaesthesia over the left thigh and anaesthesia below the knees bilaterally, together with areflexia in both lower extremities. Decompressive laminectomies of vertebrae L1-5 were performed. Postoperatively there was good recovery of motor function, but he still had bladder emptying difficulty and had a paralysed anal sphincter with faecal incontinence. He was transferred to our centre 5 weeks postsurgery. There was decreased sensation to pinprick and light touch below L5 bilaterally. His motor score was 38 in the right and 32 in the left side.

During the rehabilitation process he made considerable progress in walking, and on discharge his motor score improved to 42 in the right and 38 in the left side. He was able to walk with a left ankle foot orthosis and one elbow crutch. IV urography showed double ureters on the left side but normally functioning kidneys, and urodynamic studies revealed a functional infravesical obstruction. Treatment with prazosin and afterwards diazepam was started, and on discharge he was able to void by abdominal straining with a residual urine of less than $50 \mathrm{ml}$. His faecal incontinence continued. Regarding sexual function erection and ejaculation were weaker than normal.

\section{Case 3}

A 27 year old man suffering from achondroplasia and incomplete paraplegia was admitted to the centre. His mother and brother also had achondroplasia. Seven weeks before admission he jumped out from a second floor. Fractures of vertebrae L1 and L2 and spinal stenosis at vertebra $\mathrm{Ll}$ were verified by $\mathrm{CT}$ scan. There was decreased sensation to pinprick and light touch below L2 in both lower extremities. His motor score was 36 in the right and 44 in the left side. One day after the injury a decompressive laminectomy of vertebra $\mathrm{L} 1$ and a hemilaminectomy of vertebra $\mathrm{L} 2$ together with Harrington Luque instrumentation were performed. His neurolgical condition worsened after the operation: he complained of burning pain in both legs, muscle strength decreased in both lower extremities (motor scores right/left $32 / 40$ ) and there was urinary retention making intermittent catheterisation necessary. Muscle training was initiated and 7 weeks after the operation he was transferred to our centre. Upon admission he could walk in parallel bars, was able to void by abdominal straining with a residual urine of $100 \mathrm{ml}$ and his bowel and sexual functions were normal. Besides the typical achondroplastic deformities hypermobility of the hip joints was present. Sensation to pinprick and light touch was normal over the lower extremities. Motor scores right/left were 37/44. Both legs were hypotonic and areflexic.

During the rehabilitation process his motor function improved (motor scores right/left $42 / 48$ ) and on discharge he was able to walk with one elbow crutch and voided normally. His bowel function was normal and he had no sexual problems.

\section{Discussion}

The neurological complications of thoracolumbar stenosis in achondroplastic patients tend to become manifest clinically in adulthood. This delay in the development of neural problems suggests that the diminished size of the spinal canal is not sufficient in itself to explain these complications. Additional contributing factors to neural symptoms are age related such as herniation of the nucleus pulposus, degenerative spurring of the vertebral facets, vertebral malalignment, vertebral instability, increasing lumbar lordosis, progressive thoracolumbar kyphosis, congenital bone deformities and hypertrophy of the ligamentum flavum. . $^{13.4,6}$ During infancy the most frequent finding related to the axial skeleton is a mild thoracolumbar kyphosis. This has been attributed to hypotonia and the great majority of these curves recover with weight bearing and ambulation. When an upright posture and walking are achieved lumbar lordosis and prominence of the buttocks increase. In $23-30 \%$ persistent kyphosis develops and $36 \%$ of these curves becomes severe. The development of kyphosis has been associated with wedging or hypoplasia of the vertebral bodies at the thoracolumbar junction. ${ }^{1.2 .4 .6,7}$ Concerning the cases presented here, case 1 had a persistent kyphosis with wedging of the vertebral bodies at the thoracolumbar junction and vertebral malalignment together with degenerative spurring of the vertebral facets. In case 2 lumbar disc herniation could be considered as a further factor reducing the spinal canal. Case 3 had a spinal fracture which aggravated the spinal stenosis.

For the postpubertal achondroplastic patient the most common cause of serious disability is compression of the spinal cord 
and cauda equina. ${ }^{8,9}$ A variety of neurological syndromes result. Lutter and Langer $^{8}$ focused on the thoracolumbar spine in achondroplasia and distinguished four types of neurological deficits including (1) insidious paresthesiae, (2) intermittent claudication exacerbated by walking, (3) distinct nerve root compression, and (4) paraplegia. Subsequent studies have confirmed these syndromes, but emphasized that several may coexist in the same patient $^{10}$ as in cases 1 and 2 reported in this article. Paresthesiae are described as tingling, pin pricking, numbness, burning and a sleepy or dead sensation in the legs; this has been present in all of the cases here reported. They often occur after prolonged standing or walking, and are relieved by forward flexion, squatting or assuming a non weight bearing position. ${ }^{1}$ Spinal stenosis may give rise to backache and radiating pain to the leg as in case 1. Foot drop may often develop unilaterally resulting from nerve root compression ${ }^{1,9}$ as in case 2 . Occasionally these neurological complaints may have an abrupt onset following undue physical exertion as occured in case 3 , or from an accident. ${ }^{1}$

An accurate definition of the pathological relationships between the nervous structures and the parts of the skeleton that are malformed or have degenerated can be achieved by means of CT, myelo-CT and MRI. ${ }^{11}$ Myelography is difficult to perform technically in these patients. ${ }^{9}$ MRI distinguishes neural elements from the spinal fluid; and direct axial, sagittal, and coronal imaging are possible. On the other hand, spatial resolution is superior with $\mathrm{CT}$, and cortical bone and calcifications are not well seen by MRI. ${ }^{11}$

Treatment of thoracolumbar stenosis in achondroplasia varies according to the anatomopathological alterations of the spine and the clinical status. Surgical decompression by means of laminectomy is often recommended and considered to be an effective treatment for spinal stenosis if performed early in the course of the neurological syndrome. ${ }^{9,10}$ When spinal stenosis is combined with thoracolumbar kyphosis prognosis is less favorable than in those who have spinal stenosis without kyphosis and with or without disc herniation. Patients with severe neurological damage appear to have a less favourable prognosis. ${ }^{3,8,12,13} \mathrm{Al}$ though rehabilitation of spinal cord injured patients who have previously been suffering from neurological, orthopaedic and/or congenital disorders has been described as being much more complicated, ${ }^{14}$ a considerable improvement was obtained during the rehabilitation process for the three patients described in this article.

\section{Acknowledgement}

The Department of Radiology, Hvidovre Hospital, University of Copenhagen, Denmark are thanked for permission to use the CT-scan picture presented in Figure 1.

\section{References}

1 Scott CJ (1976) Achondroplastic and hypochondroplastic dwarfism. Clin Orthop 114: 19-28.

2 Davies RW, Walsh WK, Gormley J (1981) Achondroplasia and hypochondroplasia. J Bone Joint Surg 63B: 508-515.

3 Ferrante L, Acqui M, Mastronardi L, Celli P, Fortuna A (1991) Stenosis of the spinal canal in achondroplasia. Italian J Neurol Sci 12: 371-375.

4 Bethem D, Winter RB, Lutter L, Moe JH, Bradford DS, Lonstein JE, et al (1981) Spinal disorders of dwarfism. J Bone Joint Surg 63A: 1412-1425.

5 Ditunno JF (1992) Standards for Neurological and Functional Classification of Spinal Cord Injury. 4th ed. ASIA, Chicago, Illinois: 1-26.

6 Kopits SE (1976) Orthopedic complications of dwarfism. Clin Orthop 114: 153-179.

7 Goldberg MJ (1976) Orthopedic aspects of bone dysplasias. Orthop Clin North Am 7: 445-456.

8 Lutter LD, Langer LO (1977) Neurological symptoms in achondroplastic dwarfs-surgical treatment. $J$ Bone Joint Surg 59A: 87-92.

9 Pyeritz RE, Sack GH, Udvarhelyi GB (1987) Thoracolumbosacral laminectomy in achondroplasia: Long term results in 22 patients. Am J Med Genet 28: 433-444.

10 Morgan DF, Young RF (1980) Spinal neurological complications of achondroplasia. Results of surgical treatment. J Neurosurg 52(4): 463-472. 
11 Fortuna A, Ferrante L, Acqui M, Santoro A, Mastronardi L (1989) Narrowing of thoraco-lumbar spinal canal in achondroplasia. J Neurol Sci 33: 188-196.

12 Pyeritz RE, Sack GH, Udvarhelyi GB (1980) Surgical intervention in achondroplasia. Johns Hopkins Med J 146: 203-209.

13 Shikata J, Yamamuro T, Iida H, Kono H, Mori E (1988) Surgical treatment of achondroplastic dwarfs with paraplegia. Surg Neurol 29: 125-130.

14 Ohry A, Frankel HL (1984) Rehabilitation after spinal cord injuries complicated by previous lesions. Paraplegia 22: 291-296. 